\title{
Erratum to: When errors count: an EEG study on numerical error monitoring under performance pressure
}

\author{
Frieder L. Schillinger ${ }^{1,2} \cdot$ Bert De Smedt ${ }^{3} \cdot$ Roland H. Grabner $^{2}$
}

Published online: 1 April 2016

(C) FIZ Karlsruhe 2016

\section{Erratum to: ZDM Mathematics Education DOI 10.1007/s11858-015-0746-8}

The authors very much regret that errors have slipped into their contribution to the special issue on cognitive neuroscience. In the text, the terms "lower" and "reduced" were used to describe impaired response times in the high pressure condition (as compared to the low pressure condition) instead of the correct terms "higher" or "increased".

The corrections are listed below:

Keywords Choking under pressure · Test anxiety $\cdot$ Response monitoring $\cdot$ Error-related negativity $(\mathrm{ERN})$

\subsection{Hypotheses}

The next to last sentence should read:

At the behavioral level, we expected, firstly, that response times are higher in the high pressure condition relative to the low pressure condition.

The online version of the original article can be found under doi:10.1007/s11858-015-0746-8.

Frieder L. Schillinger

frieder.schillinger@uni-graz.at

1 Georg-Elias-Müller-Institute of Psychology, University of Göttingen, Waldweg 26, 37073 Göttingen, Germany

2 Department of Psychology, University of Graz, Universitätsplatz 2, 8010 Graz, Austria

3 Faculty of Psychology and Educational Sciences, University of Leuven, Leopold Vanderkelenstraat 32, Box 3765,

3000 Leuven, Belgium

\subsection{Behavioral data}

In the second paragraph the next to last sentence should read:

Confirming our hypothesis, response times were significantly increased when performance pressure was applied, $F(1,17)=4.79, p=.041$.

\section{Discussion}

The second paragraph should read:

Regarding the ERP analyses, we hypothesized that the ERN and/or the $\triangle E R N$ are increased by both performance pressure and test anxiety. At the behavioral level, we expected, firstly, that response times are higher in the high pressure condition relative to the low pressure condition. Secondly, we predicted response times to linearly increase with the individual level of test anxiety.

The beginning of the fifth paragraph should read:

Despite the differences in electrophysiological measures, behavioral data provided mixed evidence in regard of our hypotheses. As expected, response times were higher in the high pressure condition than in the low pressure condition. 